\title{
Fluidoterapia para la Prevención de Hipotensión Arterial Secundaria a Anestesia Espinal en Operación Cesárea: ¿Tenemos todas las respuestas?
}

\author{
Fluid Therapy Preventing Arterial Hypotension after Spinal \\ Anesthesia for Cesarean Section: \\ Do we have all the answers?
}

DAVOR MIRANDA ${ }^{1}$, HECTOR J. LACASSIE ${ }^{2}$

\begin{abstract}
Spinal anesthesia is the technique of choice for patients undergoing cesarean section. One of the most common adverse effects of this technique is arterial hypotension, which if severe, can result in serious maternal and fetal injury or death. Different alternatives exist to prevent and treat hypotension associated to spinal anesthesia; one of them is fluid therapy. The objective of this review is to evaluate the currently available evidence for different fluid therapy alternatives and assess their effectiveness. Nowadays, it does not seem advisable to use preload with crystalloids as evidence suggests its ineffectiveness. As for the other three alternatives (preload with colloids, coload with crystalloids and coload with colloids) there is no superiority among them and neither of them seems effective enough to be used as monotherapy. Due to its safety and ease of use, coload with crystalloids associated to alpha agonist vasopressor therapy seems to be the best management strategy. Further studies should aim at the best way to administer fluids in order to optimize vasopressor therapy.
\end{abstract}

\section{RESUMEN}

La técnica anestésica de elección para operación cesárea es la anestesia subaracnoídea. Uno de los principales efectos adversos de esta técnica es la hipotensión arterial, que puede llegar a ocasionar graves complicaciones. Existen diversas alternativas para la prevención y manejo de la hipotensión arterial; una de ellas la fluidoterapia. El objetivo de esta revisión es evaluar la evidencia reciente disponible para las distintas alternativas de fluidoterapia y evaluar su efectividad. Actualmente no parece recomendable el utilizar precarga con cristaloides para
Key words: Anesthesia, spinal, cesarean section, hypotension, isotonic solutions.

Palabras clave: Anestesia, espinal, cesárea, hipotensión arterial, soluciones isotónicas.

División de Anestesiología, Facultad de Medicina, Pontificia Universidad Católica de Chile.

Alumno Interno de Medicina.

Profesor Asociado en Anestesiología.

Correspondencia a:

Héctor J. Lacassie

E-mail. lacassie@med.puc.cl

Marcoleta 377, $4^{\circ}$ piso, Dpto. Anestesiología, Hospital Clínico UC, Santiago, Chile. Teléfono: +569-22-354-3270. 
la prevención de hipotensión arterial. En cuanto a las otras tres alternativas (precarga con coloides, cocarga con cristaloides o cocarga con coloides) ninguna parece ser más efectiva que las otras previniendo hipotensión arterial y ninguna sería efectiva como monoterapia. Por su disponibilidad y seguridad de uso, al día de hoy la cocarga con cristaloides asociada a terapia vasopresora alfa agonista parece ser la mejor alternativa. Estudios futuros debieran apuntar a encontrar la forma de infusión que optimize la terapia con vasopresores.

\section{Introducción}

a anestesia subaracnoídea o espinal es actualmente la técnica de elección para operación cesárea debido a que es un método simple, confiable, rápido, costo efectivo y además permite el apego inicial por parte de la madre con el recién nacido. Sin embargo no está exenta de complicaciones. Una de las más frecuentes es la hipotensión arterial, en hasta $80 \%{ }^{1,2}$. Las complicaciones de la hipotensión arterial van desde síntomas como náuseas y vómitos hasta hipoperfusión tisular y complicaciones neonatales secundarias a una disminución en el flujo uteroplacentario en los casos más graves ${ }^{3}$. Debido a la importancia que puede llegar a tener es relevante su prevención. Se han utilizado distintas técnicas con este fin que incluyen: estrategias mecánicas, uso de fármacos y fluidoterapia 4 . El objetivo de esta revisión es evaluar la evidencia reciente disponible con respecto a las diferentes alternativas de fluidoterapia y si existe alguna superior a las demás en la prevención de hipotensión arterial o en los otros desenlaces clínicos.

\section{Alternativas de fluidoterapia}

Existen diferentes variables que se pueden manipular en cuanto a la fluidoterapia y estas son: volumen y velocidad de infusión, tipo de fluido y el momento en que se administre. Así, tendremos la posibilidad de utilizar cristaloides o coloides y en cuanto a la temporalidad, de iniciar la fluidoterapia antes de la anestesia (precarga) o concomitantemente con ella (cocarga). De esto se desprenden cuatro alternativas posibles: precarga con cristaloides, precarga con coloides, cocarga con cristaloides y cocarga con coloides.

\section{Precarga con cristaloides}

La precarga con cristaloides (cloruro de sodio ( $\mathrm{NaCl})$ 0,9\% o ringer lactato $(\mathrm{RL})$ ) fue el primer intento de utilizar fluidoterapia para prevenir la hipotensión arterial secundaria a la anestesia espinal en operación cesárea. Rout y cols., demostraron que la disminución de la hipotensión no era clínicamente significativa al utilizar $20 \mathrm{ml} / \mathrm{kg}$ versus sin fluidoterapia (incidencia de hipotensión arterial: $55 \%$ vs $71 \%$ respectivamente, $p$ $=n s)^{5}$. Otros estudios han intentado usar diferentes volúmenes, sin lograr mejores resultados ${ }^{6,7}$. Hoy se reconoce que la precarga con cristaloides no es un método efectivo para disminuir la hipotensión arterial en estas condiciones, menos aún comparado con las otras alternativas disponibles.

\section{Precarga con coloides}

La precarga con coloides pareciera tener un mayor efecto en la prevención de hipotensión arterial que la precarga con cristaloides ${ }^{8}$. Hace ya algunos años Riley y cols., realizaron un estudio aleatorio donde 40 mujeres recibieron $500 \mathrm{ml}$ de coloide (hidroxi etil almidón (HES) $6 \%$ ) + $1.000 \mathrm{ml}$ de RL versus $2.000 \mathrm{ml}$ de RL. El grupo HES tuvo una incidencia de hipotensión arterial de $45 \%$ vs $85 \%$ ( $p=0,019)$ en el grupo sólo con cristaloides ${ }^{9}$. Tamilselvan y cols., compararon el efecto de precarga de $1.500 \mathrm{ml}$ de cristaloides, $500 \mathrm{ml}$ de HES y $1.000 \mathrm{ml}$ de HES sobre el gasto cardiaco medido con ecografía doppler. El grupo que recibió $1.000 \mathrm{ml}$ de HES fue el único que mantuvo el aumento en el gasto cardíaco generado inicialmente en todos los grupos por la infusión de volumen ${ }^{10}$.

Una de las grandes incertidumbres que se ha tenido con el uso de coloides ha sido su perfil de seguridad. Recientemente se realizó el protocolo CAESAR, un estudio aleatorio y multicéntrico cuyo objetivo era determinar la superioridad y seguridad de HES $6 \%$ por sobre RL. El grupo que recibió HES 6\% + RL mostró menor hipotensión e hipotensión sintomática (mareos, náuseas/vómitos o ambos) que el grupo sólo con $\mathrm{RL}(36,6 \%$ vs $55,3 \%$ valor $\mathrm{p}$ de una cola $=0,025$ y $3,7 \%$ vs $14,1 \%$ respectivamente, $p=0,028)$, sin demostrar diferencias en el consumo de vasopresores (fenilefrina) ni en los desenlaces perinatales. Como parte del estudio se evaluaron muestras de sangre en busca de presencia de HES en el recién nacido, no en- 
contrándose evidencia de traspaso transplacentario ${ }^{11}$. Una revisión sistemática posterior realizada por Ripolles y cols., apoyaría la superioridad de los coloides por sobre los cristaloides para prevención de hipotensión arterial, independiente del momento de la administración de ellos (RR [IC95\%]: 0,70 [0,53-0,92], p=0,01), aunque no así para hipotensión arterial sintomática (RR [IC95\%] 0,75 [0,41-1,38]; $p=0,33)^{12}$. Cabe señalar que gran parte de las pacientes de todas formas requirieron apoyo con vasopresores y no hubo diferencias significativas en la dosis de fenilefrina utilizada.

\section{Cocarga con cristaloides}

Se sospecha que la razón por la que la precarga con cristaloides es poco efectiva es por el poco tiempo que esta permanece en el intravascular. Es por esto que se pensó en iniciar la administración de volumen concomitantemente con el inicio de la anestesia. Dyer y cols., realizaron un estudio aleatorio con 50 embarazadas donde las pacientes recibieron $20 \mathrm{ml} / \mathrm{Kg}$ de RL. Un grupo lo recibió 20 minutos antes de la inducción y otro inmediatamente después del inicio de la anestesia, disminuyendo considerablemente el porcentaje de hipotensión arterial que requiriera drogas vasoactivas $(60 \% \text { vs } 36 \%, p=0,047)^{13}$. Un reciente estudio prospectivo, controlado y aleatorio confirmó que la cocarga con cristaloides es más efectiva que la precarga con el mismo, disminuyendo la incidencia de hipotensión arterial $(53 \%$ vs $83 \%, p=0,026)$ y la dosis necesaria de drogas vasoactivas, en este caso, efedrina $(7,5 \mathrm{mg} \text { vs } 15 \mathrm{mg}, p=0,015)^{14}$. A pesar de que la cocarga con cristaloides parece ser más efectiva que la precarga, los autores concuerdan en que por sí misma no basta para un adecuado manejo de la presión arterial. En otro estudio aleatorio y controlado realizado por Tawfik y cols., no encontraron diferencias significativas entre utilizar cocarga de cristaloi- des ( $1.000 \mathrm{ml}$ de RL) o precarga de coloides $(500 \mathrm{ml}$ de HES) para hipotensión ni para hipotensión grave $(42,2 \%$ vs $52,4 \% p=0,18$ y $9,8 \%$ vs $15,5 \% p=0,31$ respectivamente) $)^{15}$, lo que habla positivamente sobre la cocarga con cristaloides al ser al menos equivalente en efectividad a una precarga con coloides, sin sumar los riesgos de esta última.

\section{Cocarga con coloides}

Se intentó determinar si las ventajas de la cocarga versus precarga de cristaloides se aplicaban también a los coloides. Diversos estudios compararon la efectividad de la cocarga versus precarga con coloides, sin lograr encontrar diferencias significativas ${ }^{16-19}$. Esto tendría sentido considerando que la teoría detrás de la inefectividad de los cristaloides como precarga estaría determinada por su baja permanencia en el espacio intravascular, lo que no ocurría con los coloides. Por el momento, el estudio de Mcdonald y cols., sigue siendo el único protocolo donde se compara cocarga de cristaloides versus cocarga de coloides, en el cual no se logró encontrar una diferencia significativa en el desenlace primario del estudio que fue gasto cardiaco medido por ecografía doppler. Además, no hubo diferencias en los requerimientos de vasopresores o en la estabilidad hemodinámica, por lo que concluyen que el uso de coloides no ofrece ventajas sobre los cristaloides cuando son utilizados en conjunto con una infusión de fenilefrina durante una anestesia espinal para cesárea electiva ${ }^{20}$.

\section{Discusión}

En base a las diferentes combinaciones posibles en cuanto al momento de infusión, existen 4 opciones del manejo de la fluidoterapia descritas hasta ahora. La evidencia parece indicar que la precarga con cris-

\begin{tabular}{|c|c|c|c|c|c|}
\hline & Control & CR precarga & CR cocarga & COL precarga & COL cocarga \\
\hline Control & & Inefectivo $^{5}$ & $C R>$ control $^{22}$ & $\mathrm{COL}>$ Control $^{16}$ & $\mathrm{COL}>$ Control $^{16}$ \\
\hline CR precarga & Inefectivo 5 & & CO $>$ pre $\mathrm{pr}^{13,14}$ & $\mathrm{COL}>\mathrm{CR}^{8-11}$ & Sin info \\
\hline CR cocarga & $C R>$ control $^{22}$ & co $>$ pre ${ }^{13,14}$ & & Equivalentes $^{15}$ & Equivalentes ${ }^{20}$ \\
\hline COL precarga & $\mathrm{COL}>$ Control $^{16}$ & $\mathrm{COL}>\mathrm{CR}^{8-11}$ & Equivalentes ${ }^{15}$ & & Equivalentes ${ }^{16-20}$ \\
\hline COL cocarga & $\mathrm{COL}>$ Control $^{16}$ & Sin info & Equivalentes ${ }^{20}$ & Equivalentes ${ }^{16-20}$ & \\
\hline
\end{tabular}

CR: cristaolide; COL: coloide; co: cocarga; pre: precarga; Sin info: sin estudios que comparen contra placebo o sin tratamiento. 
taloides es inferior a las otras tres en la prevención de hipotensión arterial post anestesia espinal en cesárea. Entre las otras tres alternativas (precarga con coloides, cocarga con cristaloides y cocarga con coloides) no se ha podido demostrar de forma concluyente que alguna de ellas sea superior a las otras en la prevención de hipotensión arterial. El resumen de la magnitud del efecto de las distintas alternativas de fluidoterapia se puede observar en la Tabla 2. La dispersión de los datos se debe a múltiples factores que difieren entre los distintos estudios: volumen de infusión, drogas vasoactivas utilizadas concomitantemente, población estudiada y otros. A pesar de esto, ninguna de las alternativas califica como posible monoterapia para el manejo de hipotensión arterial.

Un elemento importante a recalcar es que no parece del todo precisa la conclusión que el momento de infusión (precarga versus cocarga) no tenga relevancia clínica. Esto impresiona ser cierto para la comparación con coloides, más no en cuanto a la comparación con cristaloides. En el metanálisis de Banerjee y cols. ${ }^{21}$, a pesar de realizar un análisis por separado de cristaloides y coloides, describen las múltiples limitaciones de ese metanálisis que incluyen: ser estudios pequeños, muchos sin ciego, volumen variable utilizado en cada estudio y uso de diferentes vasopresores. De hecho, sólo uno de los estudios incluidos tenía significancia estadística y correspondía a aquel que utilizó el mayor volumen de cristaloides $(20 \mathrm{ml} /$ $\mathrm{kg})^{13}$. Ahora bien, en base a los resultados obtenidos en los diversos estudios, si realmente existiera una diferencia estadísticamente significativa en la incidencia de hipotensión entre precarga y cocarga, esta sería probablemente pequeña por lo que se requeriría un gran número de sujetos en cada rama del estudio para poder detectarla y clínicamente parece ser poco importante.

La mayoría de las revisiones antes mencionadas no incluyen estudios como el de Ngan Kee y cols., en el cual se compara un grupo con y otro sin cocarga de cristaloides asociado a fenilefrina profiláctica. En este se observó una reducción significativa en la incidencia de hipotensión arterial en el grupo que recibió cocarga con cristaloides versus aquel que no ( $28 \%$ vs $2 \%$, $\mathrm{p}<0,001)^{22}$. Es importante evaluar la fluidoterapia en este contexto, que se acerca más a lo que es la práctica clínica en la actualidad. Al día de hoy, no parece haber duda que la mejor alternativa de prevención de hipotensión arterial es el uso de vasopresores alfa adrenérgicos, asociado a algún tipo de cocarga de fluidos. Aún queda por definir si la forma de administración juega algún rol en la prevención de hipotensión en este contexto y si existe algún método que optimice el tratamiento con vasopresores. Han surgido nuevas formas de administración de los agonistas alfa adrenérgicos como los sistemas controlados por retroalimentación en asa cerrada (closed loop feedback). En un reciente estudio, Ngan Kee y cols., evaluaron el uso de esta técnica asociado a hidratación, demostrando una buena efectividad clínica ${ }^{23}$. Aunque aún queda mucho por analizar y ajustar antes de que este método constituya el estándar de cuidado definitivo, el uso de este tipo de técnicas estandarizadas de administración de vasopresores podría ser útil para

\begin{tabular}{|c|c|c|c|c|c|}
\hline 1er autor & CR pre & COL pre & $\mathrm{Cr}$ co & COL co & valor $p$ \\
\hline Riley $^{9}$ & $85 \%$ & $45 \%$ & & & 0,019 \\
\hline Mercier $^{11^{*}}$ & $55,3 \%$ & $36,3 \%$ & & & $0,025 *$ \\
\hline Dyer ${ }^{13}$ & $60 \%$ & & $36 \%$ & & 0,047 \\
\hline $\mathrm{Oh}^{14}$ & $83 \%$ & & $53 \%$ & & 0,026 \\
\hline Tawfik ${ }^{15}$ & & $52,4 \%$ & $42,2 \%$ & & NS \\
\hline Nishikawa ${ }^{16}$ & & $11,1 \%$ & & $16,7 \%$ & NS \\
\hline Carvalho ${ }^{17}$ & & $48 \%$ & & $30 \%$ & NS \\
\hline Siddik-Sayyid ${ }^{18}$ & & $68 \%$ & & $75 \%$ & NS \\
\hline Mcdonald ${ }^{20}$ & & & $60 \%$ & $40 \%$ & NS \\
\hline Banerjee $^{21}$ & \multicolumn{2}{|c|}{$62,4 \%$} & \multicolumn{2}{|c|}{$59,3 \%$} & NS \\
\hline Ripolles ${ }^{12 \#}$ & $61,2 \%$ & $45,6 \%$ & $61,2 \%$ & $45,6 \%$ & 0,03 \\
\hline
\end{tabular}

CR: cristaloides; COL: coloides; pre: precarga; co: cocarga. *valor p de una cola; \#agrupados cristaloides en pre y cocarga con coloides en pre y cocarga; NS: no significativo. 
encontrar también el verdadero rol de la fluidoterapia en la prevención de hipotensión.

Otro aspecto importante a considerar es la seguridad de los cristaloides y coloides. Últimamente han aparecido revisiones comparando cristaloides con coloides en otros ámbitos que parecieran apoyar la teoría de que los coloides no están exentos de efectos adversos, como falla renal y necesidad de diálisis ${ }^{24}$. Una revisión sistemática que comparaba diversos coloides versus cristaloides observó una consistente alteración de la coagulación medida con tromboelastograma y una tendencia a mayor sangrado perioperatorio en el grupo de coloides ${ }^{25}$. A pesar de que el estudio CAESAR tenía dentro de sus objetivos el evaluar la seguridad del HES 6\% como fluidoterapia, el número de pacientes reclutadas sólo fue el necesario para detectar el desenlace de hipotensión arterial, no así los otros efectos adversos ${ }^{11}$.

Un último elemento a considerar es que la mayoría de los estudios se realizan en mujeres sanas, por lo que el considerar la capacidad de la paciente de tolerar los volúmenes infundidos y posibles alteraciones en la homeostasis queda en manos del clínico. La validez externa para poblaciones de alto riesgo no está asegurada. Además, la rigurosidad en los criterios para seleccionar o excluir a las diferentes pacientes varía entre los diferentes estudios, por lo que podrían haber otras variables que influyeran en la heterogeneidad de los resultados obtenidos.

En conclusión, el uso de cocarga con cristaloides asociada a un vasopresor alfa agonista en forma de bolos o infusión, sigue siendo la alternativa con mejor relación riesgo/beneficio para el tratamiento contra hipotensión arterial en operación cesárea bajo anestesia espinal. El costo y los riesgos asociados al uso de coloides aún superan su potencial beneficio. Queda aún pendiente evaluar en futuros estudios, cuál es el volumen óptimo de fluídos para complementar a las drogas vasoactivas en la prevención de hipotensión o si el método de administración es el que hará una diferencia clínica significativa. El uso de nuevas tecnologías podrían ser útiles en un futuro próximo tanto para el manejo de la hipotensión arterial, como para dilucidar el verdadero rol de la fluidoterapia.

\section{Referencias}

1. Riley ET, Cohen SE, Macario A, Desai JB, Ratner EF. Spinal versus epidural anesthesia for cesarean section: a comparison of time efficiency, costs, charges, and complications. Anesth Analg 1995 Apr;80(4):709-12.

2. Rocke DA, Rout CC. Volume preloading, spinal hypotension and caesarean section. Br J Anaesth 1995 Sep;75(3):257-9.

3. Maayan-Metzger A, SchushanEisen I, Todris L, Etchin A, Kuint J. Maternal hypotension during elective cesarean section and short-term neonatal outcome. Am J Obstet Gynecol 2010 Jan;202(1):56.e1-5.

4. Arias J, Lacassie HJ. [Prophylaxis and treatment of arterial hypotension during caesarean with spinal anaesthesia]. Rev Esp Anestesiol Reanim 2013 Nov;60(9):511-8.

5. Rout CC, Rocke DA, Levin J, Gouws E, Reddy D. A reevaluation of the role of crystalloid preload in the prevention of hy- potension associated with spinal anesthesia for elective cesarean section. Anesthesiology 1993 Aug;79(2):262-9.

6. Park GE, Hauch MA, Curlin F, Datta S, Bader AM. The effects of varying volumes of crystalloid administration before cesarean delivery on maternal hemodynamics and colloid osmotic pressure. Anesth Analg 1996 Aug;83(2):299-303.

7. Morgan PJ, Halpern SH, Tarshis $J$. The effects of an increase of central blood volume before spinal anesthesia for cesarean delivery: a qualitative systematic review. Anesth Analg 2001 Apr;92(4):997-1005.

8. Ueyama $\mathrm{H}, \mathrm{He} \mathrm{YL}$, Tanigami $\mathrm{H}$, Mashimo T, Yoshiya I. Effects of crystalloid and colloid preload on blood volume in the parturient undergoing spinal anesthesia for elective Cesarean section. Anesthesiology 1999 Dec;91(6):1571-6.

9. Riley ET, Cohen SE, Rubenstein AJ, Flanagan B. Prevention of hypotension after spinal anesthe- sia for cesarean section: six percent hetastarch versus lactated Ringer's solution. Anesth Analg 1995 Oct;81(4):838-42.

10. Tamilselvan $P$, Fernando R, Bray J, Sodhi M, Columb M. The effects of crystalloid and colloid preload on cardiac output in the parturient undergoing planned cesarean delivery under spinal anesthesia: a randomized trial. Anesth Analg 2009 Dec;109(6):1916-21.

11. Mercier FJ, Diemunsch $P$, Ducloy-Bouthors AS, Mignon A, Fischler M, Malinovsky JM et al.; CAESAR Working Group. 6\% Hydroxyethyl starch (130/0.4) vs Ringer's lactate preloading before spinal anaesthesia for Caesarean delivery: the randomized, double-blind, multicentre CAESAR trial. Br J Anaesth 2014 Sep;113(3):459-67.

12. Ripollés Melchor J, Espinosa Á, Martínez Hurtado E, Casans Francés R, Navarro Pérez R, Abad Gurumeta $A$ et al. Colloids versus crystalloids in the prevention of hypotension induced by spinal 
anesthesia in elective cesarean section. A systematic review and meta-analysis. Minerva Anestesiol 2015 Sep;81(9):1019-30.

13. Dyer RA, Farina $Z$, Joubert IA, Du Toit P, Meyer M, Torr G et al. Crystalloid preload versus rapid crystalloid administration after induction of spinal anaesthesia (coload) for elective caesarean section. Anaesth Intensive Care 2004 Jun;32(3):351-7.

14. Oh AY, Hwang JW, Song IA, Kim $\mathrm{MH}$, Ryu JH, Park HP et al. Influence of the timing of administration of crystalloid on maternal hypotension during spinal anesthesia for cesarean delivery: preload versus coload. BMC Anesthesiol 2014 May; 14(1):36.

15. Tawfik MM, Hayes SM, Jacoub FY, Badran BA, Gohar FM, Shabana AM et al. Comparison between colloid preload and crystalloid co-load in cesarean section under spinal anesthesia: a randomized controlled trial. Int J Obstet Anesth 2014 Nov;23(4):317-23.

16. Nishikawa K, Yokoyama N, Saito S, Goto F. Comparison of effects of rapid colloid loading before and after spinal anesthesia on maternal hemodynamics and neonatal outcomes in cesarean section. J Clin Monit Comput
2007 Apr;21(2):125-9.

17. Carvalho B, Mercier FJ, Riley ET, Brummel C, Cohen SE. Hetastarch co-loading is as effective as pre-loading for the prevention of hypotension following spinal anesthesia for cesarean delivery. Int J Obstet Anesth 2009 Apr;18(2):150-5.

18. Siddik-Sayyid SM, Nasr VG, Taha SK, Zbeide RA, Shehade JM, Al Alami AA et al. A randomized trial comparing colloid preload to coload during spinal anesthesia for elective cesarean delivery. Anesth Analg 2009 Oct;109(4):1219-24.

19. Teoh WH, Sia AT. Colloid preload versus coload for spinal anesthesia for cesarean delivery: the effects on maternal cardiac output. Anesth Analg 2009 May;108(5):1592-8.

20. McDonald S, Fernando R, Ashpole K, Columb M. Maternal cardiac output changes after crystalloid or colloid coload following spinal anesthesia for elective cesarean delivery: a randomized controlled trial. Anesth Analg 2011 Oct;113(4):803-10.

21. Banerjee $A$, Stocche RM, Angle P, Halpern SH. Preload or coload for spinal anesthesia for elective Cesarean delivery: a metaanalysis. Can J Anaesth 2010
Jan;57(1):24-31.

22. Ngan Kee WD, Khaw KS, Ng FF. Prevention of hypotension during spinal anesthesia for cesarean delivery: an effective technique using combination phenylephrine infusion and crystalloid cohydration. Anesthesiology 2005 Oct;103(4):744-50.

23. Ngan Kee WD, Tam YH, Khaw KS, Ng FF, Lee SW. Closed-loop feedback computer-controlled phenylephrine for maintenance of blood pressure during spinal anesthesia for cesarean delivery. Anesthesia and analgesia 2017, http://Insights.ovid.com/ crossrefn $=\mathrm{fn}=00000539$ 900000000-97586: 1-7. https://doi.org/10.1213/ ANE.0000000000001974.

24. Mutter TC, Ruth CA, Dart AB. Hydroxyethyl starch (HES) versus other fluid therapies: effects on kidney function. Cochrane Database Syst Rev 2013 Jul;11(7):CD007594.

25. Rasmussen KC, Secher NH, Pedersen $\mathrm{T}$. Effect of perioperative crystalloid or colloid fluid therapy on hemorrhage, coagulation competence, and outcome: A systematic review and stratified meta-analysis. Medicine (Baltimore) 2016 Aug;95(31):e4498. 